\title{
Advanced Polymer Particles
}

\author{
Eri Yoshida, ${ }^{1}$ Takashi Kaneko, ${ }^{2}$ Toshifumi Satoh, ${ }^{3}$ \\ Yusuf Menceloglu, ${ }^{4}$ and Atsuyoshi Nakayama ${ }^{5}$
}

\author{
${ }^{1}$ Department of Environmental and Life Sciences, Toyohashi University of Technology, Toyohashi 441-8580, Japan \\ ${ }^{2}$ Graduate School of Science and Technology, Niigata University, Niigata 950-2181, Japan \\ ${ }^{3}$ Division of Biotechnology and Macromolecular Chemistry, Faculty of Engineering, Hokkaido University, Sapporo 060-8628, Japan \\ ${ }^{4}$ Faculty of Engineering and Natural Sciences, Sabanci University, 34956 Istanbul, Turkey \\ ${ }^{5}$ Health Research Institute, National Institute of Advanced Industrial Science and Technology, Osaka 563-8577, Japan
}

Correspondence should be addressed to Eri Yoshida, eyoshida@ens.tut.ac.jp

Received 27 February 2012; Accepted 27 February 2012

Copyright ( $) 2012$ Eri Yoshida et al. This is an open access article distributed under the Creative Commons Attribution License, which permits unrestricted use, distribution, and reproduction in any medium, provided the original work is properly cited.

Polymer particles support today industries based on cuttingedge technologies. The micro- and nanosized polymer particles have many applications in various fields, such as painting, printing, electronics, adhesives, paper manufacture, civil engineering and construction, cosmetics, and medical care. In these fields, the particles have played a significant role in improving the functions and performances of the materials. Examples include the rheology control of painting, improvement of the optical properties of luster, whiteness, and opacity in paper manufacture and cosmetics, strengthening concrete in civil engineering, and carriers of drug delivery systems and immunoassay in medical care. Thus, a wide variety of applications of polymer particles is based on the advantages over inorganic and metal particles in the fact that various methods can be utilized to prepare them that their size and forms can be strictly controlled over a wide range and that their surfaces and interior structures can be modified in great variety. The polymer particles have become a key material in creating new products and technologies. To accelerate the developments of new products and progress of technologies, we planned this special issue on advanced polymer particles. In this special issue, we have invited a few papers involving novel methods of preparing polymer particles and the polymers themselves, the methods that should promote such developments and progress.

One paper of this special issue addresses the fact that understanding the environmentally related unstable colloids, especially, floc structures and their formation mechanics to predict macroscopic transportation properties of the flocs are essential to engineering design using colloidal particles. The flocs dominate the dynamic properties of the unstable colloids, such as sediments in rivers, lakes, and brackish estuaries, and soils and cakes in waste water treatment processes. It is important to clarify the relationship between microscopic colloidal interactions and microscopic properties. In this study, a geometrical model explained the rate of sedimentation, rheological properties, and influence on flocculation of the colloids by water-soluble polymers.

Another paper of this special issue presents the preparation of the polymer particles by self-assembly of polymers containing cholesterol that forms thermotropic and lyotropic liquid crystallines, monolayers, micelles, and liposomes. These cholesterol-supported amphiphilic copolymers are self-assembled into different nanoordered structures depending on the copolymer structure in water through the strong stacking and hydrophobicity of the cholesterol. A following paper describes the fabrication of polymer particles with superhydrophobicity by self-assembly of random copolymers containing perfluoroalkyl chains in supercritical carbon dioxide. The copolymers formed micellar-structured spherical particles of hundreds of nanometers in diameter in a heterogeneous state at pressures lower than the cloud point pressure of $\mathrm{CO}_{2}$. The surfaces coated with the spherical particles showed superhydrophobicity with the watercontact angles of over $170^{\circ}$.

Another paper of this special issue presents the synthesis of carbazole-supporting block, random, star, and star block copolymers that self-assemble into fluorescent micelles and three-dimensional hierarchical structures. The copolymers were prepared by the controlled/living 
radical polymerizations, such as the reversible additionfragmentation chain transfer (RAFT) polymerization and atom transfer radical polymerization (ATRP). The copolymers were also functionalized with rhenium diimine complexes to serve as a photosensitizer, while the rod-coil block copolymer was employed for a white organic electroluminescence device. A paper concerns the preparation of well-defined hyperbranched polymers expected for use as molecular capsules for carriers of drug delivery systems, nanoreactors, mixture separators, viscosity modifiers, and cross-linkers to provide functional gels. The hyperbranched polymers were prepared through polymerization using a slow monomer addition method. By this slow monomer addition technique, various highly branched polymers were obtained, such as hyperbranched poly(2-hydroxymethyloxetane), polyglycerol, and glycopolymer. Other paper is on the synthesis of rod-like structured polydendrons by the polymerization of monodendron monomers having alkoxy peripheral groups using a rhodium catalyst. The membranes prepared from the polydendrons showed high oxygen permselectivity. Finally a paper introduces the application of polymer resins to photoresists for which the molecular weights of the polymer resins are required to be strictly controlled. It is reported that the use of wet ozone was effective for removal of ionimplanted resists.

Eri Yoshida

Takashi Kaneko

Toshifumi Satoh

Yusuf Menceloglu

Atsuyoshi Nakayama 

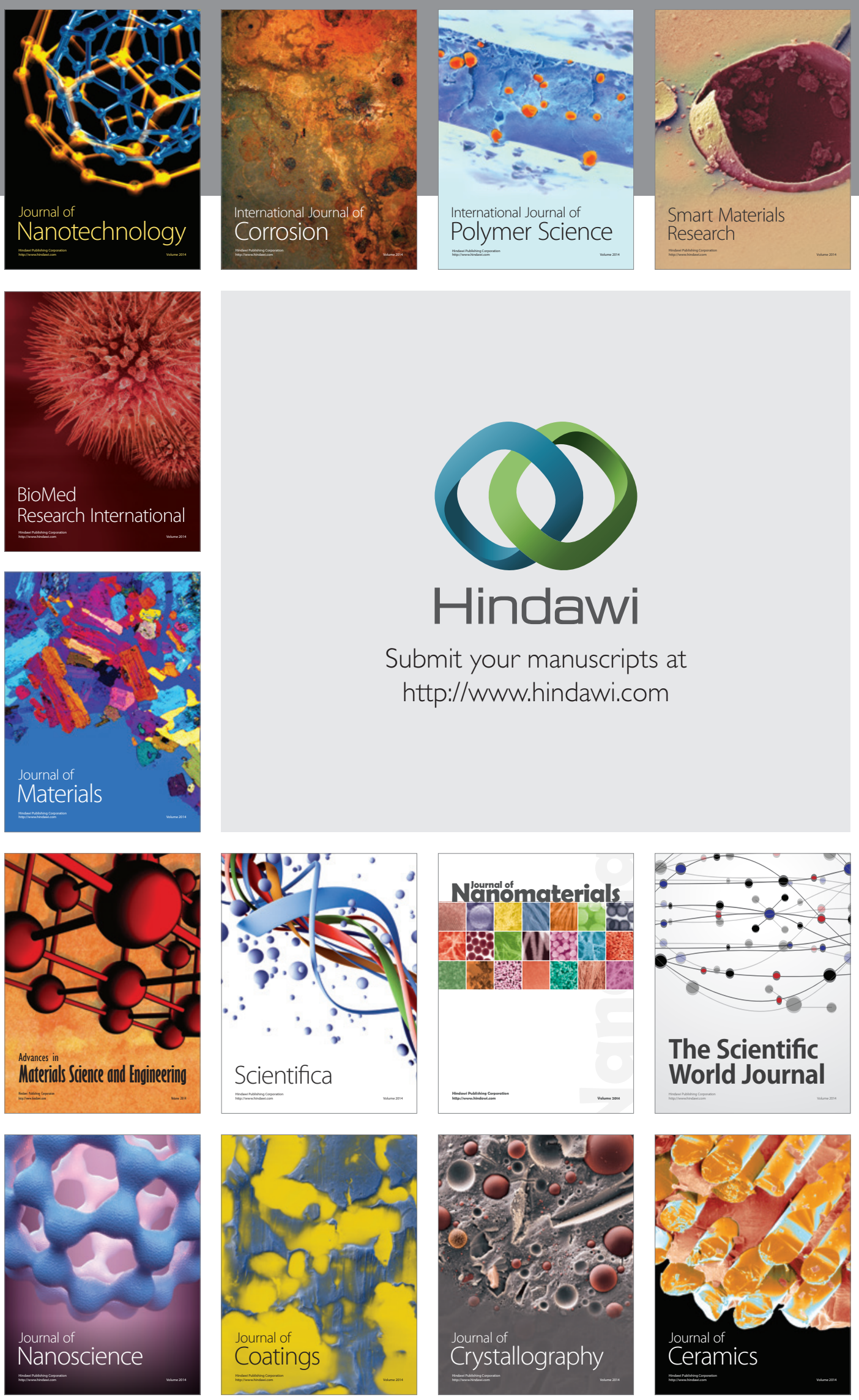

The Scientific World Journal

Submit your manuscripts at

http://www.hindawi.com

\section{World Journal}

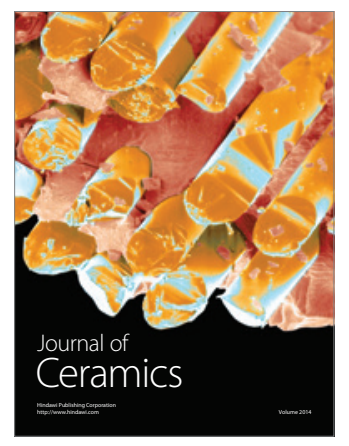

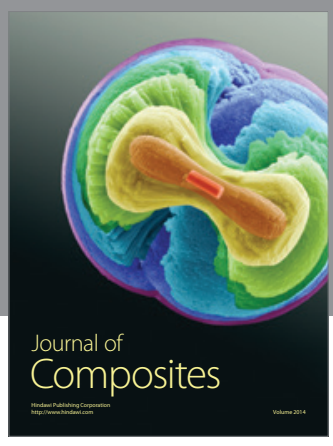
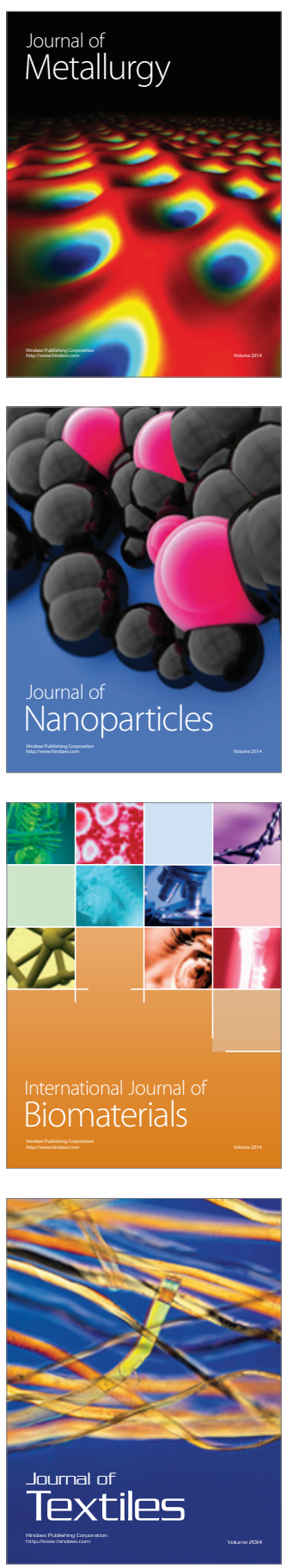